\title{
My First Phone: Smartphones Android for Kids
}

\author{
${ }^{1}$ A. Gomes, ${ }^{2}$ J. Gomes, ${ }^{3}$ A. Santos \\ Coimbra Institute of Engineering, Coimbra, Portugal \\ 1'anabela@isec.pt, ${ }^{2} a 21150324 @$ alunos.isec.pt, ${ }^{3}$ ans@isec.pt
}

Received: March 25, 2019. Revised: May 18, 2021. Accepted: October 22, 2021. Published: November 16, 2021.

\begin{abstract}
Anybody can question the advantages of a kid having and starting to use a mobile phone. Nevertheless, from a certain age parents consider the use of mobile phones by kids quite useful. The mobile phone allows an easy communication between parents and children and permits child control. Furthermore, nowadays some Android smartphones have affordable prices that can bring new technologies and other opportunities to this domain. In this paper we present a system, that includes several new easy to use applications but with a set of advanced parent control features, to adapt the phone for child use and to minimize the risks.
\end{abstract}

Keywords - Android, Smartphone, Children, Parent Control.

\section{INTRODUCTION}

$T_{\mathrm{b}}^{\mathrm{s}}$ he increasing proliferation of mobile technologies has brought forth their use by younger children. However, these technologies are not prepared for those users who, in their innocence, end up being easy targets for a wide range of dangers.

The Android platform is currently one of the most widely used on mobile devices (all over the world) [1] in a wide variety of applications and interactive games, immediately capturing the attention of users, especially the younger ones. Additionally, there are devices on the market at affordable prices making them attractive for the type of use children give them. Nevertheless, these devices provide advanced capabilities and modes of interaction more than necessary for this particular target. This raises further questions regarding the esthetic and functional suitability of these technologies, so that the interaction should be simple and intuitive. To answer these problems we thought of ways to minimize the risks of use and improve the children's interaction with these new technologies [2]. In order to provide a more adequate Android platform for use by children, some new applications were developed and others were modified.

At the same time, our intention was to include applications that enable parental control as a way to monitor not only the used applications and the communications made by the children but also to keep track of their activities and their movements.

Another objective is to try to maintain the essence of the aesthetic and functional Android platform.

The objectives of this project are to both give children a technologically evolved tool, like the Android platform, and give parents an assurance that their children do not indiscriminately communicate with unwanted people or do unsafe actions.

\section{SMARTPHONES FOR KIDS}

The tools developed in this project intended to enable a safe introduction for children to the world of new mobile technologies. To that end, a set of features must be implemented allowing the creation of a safe environment. For the achievement of such a claim, several features must exist in order to enable the following restrictions:

- send/receive SMS only for numbers that parents consider suitable;

- send/receive calls only to numbers that parents judge appropriate;

- access to the applications installed on the mobile device;

- access to the settings of the mobile device.

A mechanism to enable the child to quickly come into contact with their parents must also be provided whenever an emergency occurs. This function is intended to be as easily accessible as possible.

The system must also make the location of the device and therefore the child location available. The parents or guardian should have access to this information whenever they want. The system must also permit to keep track of multiple locations where the child has been, tracking the child's whereabouts.

In addition to the various restrictions above mentioned, and in order to better control the activities carried out by the child on his/her mobile device, all calls, messages, notes and photographs taken by the child must be recorded.

The system should also provide an application which allows children to easily create notes of various types: voice, written and pictographic. All these notes must be registered and combined into one application.

\section{STATE OF ART}

In the Google Play [3] (the main "market" where we can get Android applications) we can find applications that can help reach the main goals of this work. Presented in this section is a short description of some applications that, somehow, implement the functionalities that are intended to be included in our system. We will focus on applications that have as a main target young children.

The front end of Android devices is a type of application called launcher. This application is the hub to access all the features that we can get from these devices. There are some launchers that can be very appellative to children, because of their design and personalization capacities. Launchers like 
Apex Launcher [4], ADW.Launcher [5], Go Launcher EX [6], Nova Launcher [7], Trebuchet Launcher [8], among others. These launchers are very popular and have thousands of downloads. For instance, the Go Launcher is very popular and offers many themes, some adapted to children tastes. Nevertheless they present a lack in the control of activities that the users (children) can perform. The application Kids Place [9] is presented as a launcher but it is a normal application that restricts the child to only some applications and prohibits the use of the phone service (calls and messages). We don't want to prohibit, we just want to control and restrict the phone usage.

There are also applications whose main concern is parent control, like MMGuardian Parental Control [10], Android Parental Control [11] or App Lock [12]. MMGuardian Parental Control presents itself as an excellent parental control application. It shows, however, incompatibilities with various devices which reduces its applicability. Android Parental Control is an application that provides blocking access to applications. However, this feature also prohibits the use of the full capabilities of the Android platform, because it limits the user to the window application, not allowing a real interaction with the platform. App Lock is an application that lets you lock installed applications using a password or pattern, being very efficient and can be used with any launcher. However, we chose not to incorporate it in our system because it would increase the complexity of the settings and we wanted an easy configurable system.

Another area in which we focused was a tracker for the device location (kid location). In this category we found many applications that could support our needs, nevertheless they failed in the integration with other needs we wanted to provide. Two examples are the Missing Droid Locator [13] and the Track My Droid [14]. The application Missing Droid Locator has two important features that are: locate the phone and lock the device to protect data. The first feature is aligned with the functionality of location proposed for this system. Track My Droid is an application that has the following three main features: change sound settings depending on the location, maintain the current location and a widget for emergency aid. These applications are simply two examples, but as mentioned there are many others. The system that we will discuss in the following sections includes an integrated alternative to these applications but doesn't restrict their use as a complementary system.

Relatively to the basic features of the phone, calling and messaging, there are some applications available in the market. Phone dialer applications are not so usual but we can find a lot of applications to send and receive SMS. These applications don't present much of an advance when compared to the original ones, except at the design level, that tries to be more appealing. Examples of these SMS applications are Handcent SMS [15] and Go SMS Pro [16]. The latter application is wellintegrated with the Go Launcher, which we have talked about previously. However, it doesn't permit to configure restrictions on the numbers allowed to be used in the sending and receiving of messages.

Even though the applications available on Google Play with the features we need are diverse, generally they are not idealized for regular child use. They have quite elaborate interfaces intended for users with some experience in using this platform.

We observed that there are many applications that propose to implement some of the sought after features. However, each application implements a reduced set of characteristics in some situations or an excessive set in others. This also leads to the need of installing and configuring various applications, separately, to achieve all requirements.

\section{SYSTEM ARCHITECTURE}

As there is no tool with the intended set of features we decide to implement a new one. The generic features identified resulted in the several following applications/components: a launcher, a calling application, a messaging application, an application to create notes and an application that enables an easy configuration by the parents.

\section{A. Launcher}

As previously mentioned, one of the main objectives of this project was to shape the Android platform so that it becomes simple and intuitive for the targeted group (children). This would not be possible without the implementation of a custom launcher, from which all features can be easily reached.

The launcher was intended to be simple, intuitive and allowing quick access to the key functionalities implemented in this project: the calling application, the SMS application and the emergency calls feature. One of the important points was to control the child's access to the applications that the guardian had flagged as unsuitable for their children. The entire device is activated to avoid actions not permitted, even when the child tries to access an application through the app drawer component of the launcher.

The home screen component should derail the access and the use of widgets that have been blocked by the parents. Furthermore it must also block the addition of shortcuts to non-authorized application.

Restricting access to applications aims at the protection of the child by restricting the use of certain applications without the supervision of a guardian. This includes the internet access, too many hours of playing and the installation of unauthorized applications. Among all the available applications we could highlight some of the dangers that may arise during their use:

- Market (Play Store): It is important that this application be blocked because, through it, it is possible to easily buy/install new applications. By not blocking this application it could lead to the installation of applications with inappropriate content for children.

- System Settings: the child should be prevented from accessing settings, since having access to them he/she can perform operations that jeopardize the proper functioning of the system. 
- File Explorer: it is advisable to block this application because it allows access to the file system of the device and allows browsing, editing and deleting files, and this latter operation can affect the proper operation of some applications.

- Internet Browser: This program provides access to websites, so it is extremely important to control its use. It would be advisable that the access to websites be monitored by an adult.

- Email Application: email is now a major form of communication and it is also through this that many dangers happen. We consider it important that the child has access to an email, but this access should be supervised to ensure it is used safely.

The launcher will continue to provide the wallpaper changing function so that the child can customize the home screen wallpaper. This functionality should be accessible in the usual forms existing on the Android platform, namely: the context menu or via the launcher settings.

The launcher will have the component that enables to make calls in an emergency situation. This component will display a list of emergency contacts and each contact must be presented with a picture and its name, in order to facilitate the identification.

In order to allow quick access to the main features of the system, and since the home screen is the main interface of the Android platform, we decided to include three permanent buttons (Figure 2 - A zone) that give direct access to the emergency call function, to the regular call application and to the messaging application.

\section{B. Calling Application}

On the Calling Application the child can make their usual voice calls. This application is intended to be as simple and as understandable as possible, so that the child can learn quickly and expeditiously to interact with it. This application is also responsible for controlling the restrictions to all voice communications, allowing only outgoing calls to numbers that were authorized (configured by guardians).

\section{C.Messaging Application}

The Messaging Application will probably be the most used by the child. With this premise as a basis, it should be simple and intuitive, minimizing the effort of the child to adapt to it. This application will be responsible for actively restricting, either sending or receiving messages from contacts that are not authorized by their guardians.

\section{D.Notes Application}

The Notes Application appears from the necessity that often children have in taking notes on a particular subject, remembering a comment or expressing and fostering their creativity. With this application it is intended that the child have a way to create notes and keep track of them in an easy form.

\section{E. Settings Application}

The Settings Application enables parents to setup the other applications of this project, making use of an intuitive interface. As this application is aimed to help parents, it will list all the activities that the child performed on the platform, namely, the call $\log$, the SMS log, the notes register, the recorded photos and the location track. This application is also responsible for implementing the features that relate to the child location, provide the child's current location via SMS and makes the daily log of the places the child went to.

\section{V.DEVELOPMENT}

In this section it will be discussed the features mentioned in the previous section. To understand the structure of the system it is necessary to realize that it is divided into the following five basic applications (mentioned in the previous chapter):

- SMS Messaging Application (SMS4Kids);

- Calling Application (Phone4Kids);

- Notes Application (MyNotes);

- Settings Application (ControlCenter4kids);

- Launcher: app drawer and home screen (Launcher4Kids).

The following image (Fig. 1) shows a generic outline of the relationship between applications.

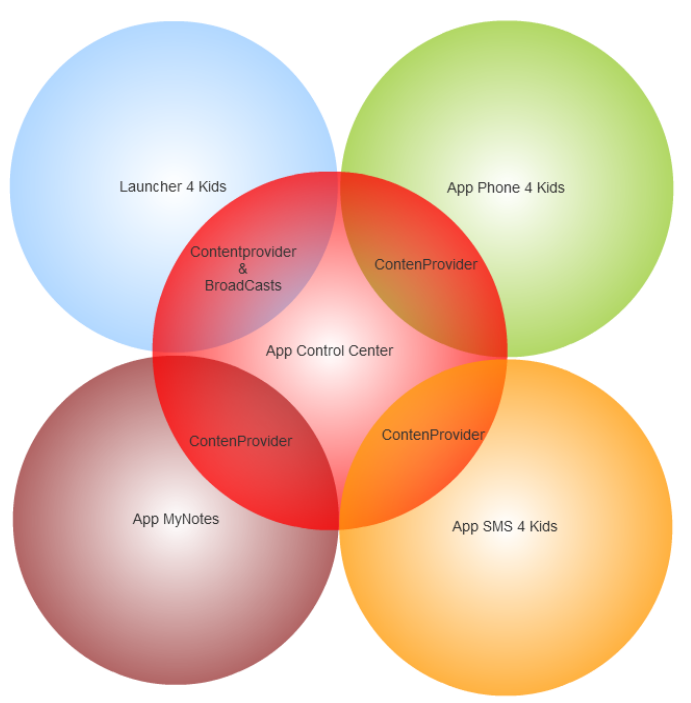

Fig. 1 My First Phone applications 


\section{A. Launcher4kids}

One of the implemented features was the launcher. The home screen of the launcher is divided into three zones (Fig. 2). The A Zone contains buttons for quick access, including the access button to the implementation of emergency call (SOS), the access button to the calling application ("Chamadas" is a portuguese word meaning Calls) and the access button to the SMS messaging application (SMS). In the $\mathrm{B}$ zone it is possible to add widgets and shortcuts for the applications. The $\mathrm{C}$ zone gives access to the app drawer, where we can view the applications by clicking on the black bar or sliding a finger over it.

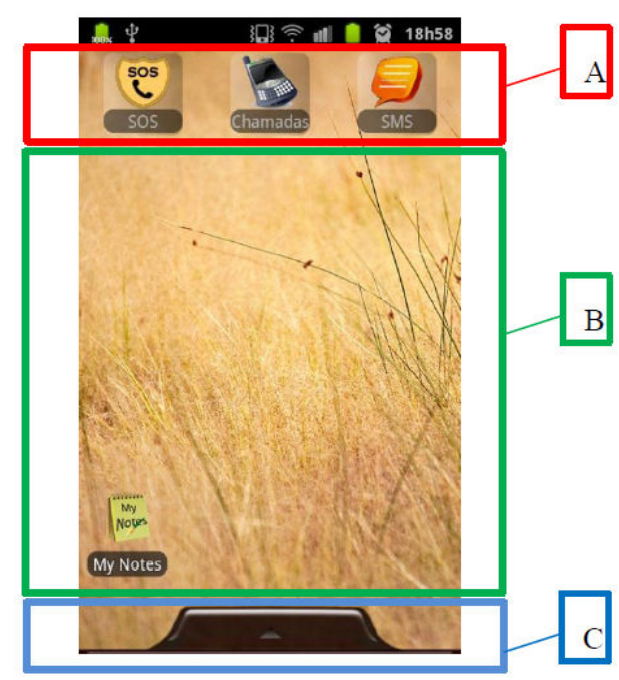

Fig. 2 Launcher4Kids Home Screen

The application of emergency calls is an activity (a component type of the Android platform characterized by having a graphical interface) embedded into the launcher. Whenever it is accessed, it connects to a content provider of the emergency contacts in the ControlCenter4Kids application in order to present a list of emergency contacts.

The launcher depends on the information that is stored in the data structures of the ControlCenter4Kids application. When it needs to know which applications are blocked, it consults the content provider of applications and the same happens when it needs to know what widgets are blocked, making a query to widgets content provider.

Another type of communication between the launcher and the application ControlCenter4Kids happens when the parents block an application and the launcher is already running. In this situation, the application sends a broadcast message to the launcher for this to block such an application.

\section{B. Phone4kids}

As mentioned before, the calling application should be as simple and intuitive as possible. For this purpose, it has two tabs at the top of the window: the call tab, that enables a call to be made, and the history tab that enables the viewing of the call $\log$. The window that allows making calls has a custom keyboard. The dialing of the number to whom the child wants to connect is possible through this area or choosing the number from a list which contains all authorized contacts. This application is responsible for reviewing all voice communications and for blocking all numbers that are not allowed. Whenever the child tries to make a call, it is checked if he/she is trying to call to an authorized number, independently of whether it is a call initialized in this calling application, or in another one installed in the system. This functionality is accomplished through registration of a broadcast receiver for outgoing calls, and the same is done for incoming calls. This application uses the contacts calls content provider (made available by the application ControlCenter4Kids) whenever we need to know which contacts are allowed to perform communications with this device. The calling application stores in a small database, the information regarding calls.

\section{C.SMS4Kids}

The messaging application is responsible for managing the sending and receiving of messages. The interface of this application is based on a message listing. When it is initialized, a list of conversations appears showing the messages that have been exchanged, with each contact. At the bottom of the window screen a button appears allowing the creation of new messages. When a conversation is selected another screen is presented, allowing the exchanged messages in that conversation to be viewed. A quick way to send a new message is available, at the bottom of the screen. This application is responsible for checking whether sent and received messages were addressed to or from authorized numbers, respectively. Like in the previous cases, whenever this application needs to know which numbers are allowed to exchange messages with, it consults the contacts content provider. It also keeps a small database of all the sent or received messages. For that purpose, this application sends information about the messages to the application ControlCenter4Kids, through a content provider.

\section{D.MyNotes}

The MyNotes application, as previously stated, intends to enable a child to create their notes easily and intuitively. It is possible to create notes of various types: voice, written and pictographic (drawings). Therefore, its interface tries to be simple and intuitive with each of its functions. This application has a small database for storing the created notes. The content of each note is stored in a file in the memory card. As in previous applications, this application is also responsible for sending information about each created note via a content provider to the ControlCenter4Kids application.

\section{E. ControlCenter4Kids}

The ControlCenter4Kids application centralizes the main system settings, enabling the configuration of all communication restrictions, the configuration of applications and the configuration of the widgets that the child will be able 
to use. It also enables the configuration of how often the child location will be updated as well as the contacts that may ask for the kid location, by SMS. In the ControlCenter4Kids there is also the option of consulting the history of the activities that the child has performed. It is, therefore, a crucial application for all the system (Fig. 3), as it provides the information to other system applications, through several content providers, so that they can perform the tasks for which they were designed.

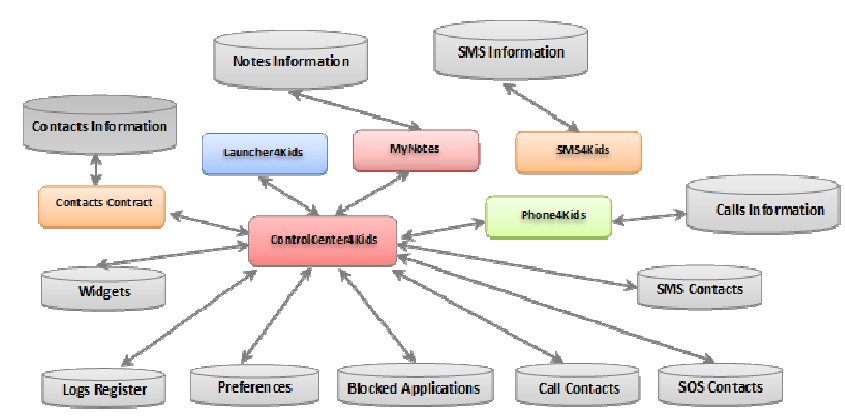

Fig. 3 Launcher4Kids Home Screen

\section{F. Contacts storage}

An important part of the information needed for the proper functioning of the system is the contact details, including the number, the name and, if possible, a photo. The latter is important so that the child can quickly identify each contact.

Since the Android platform already has a framework for managing contacts, we chose to delegate the contacts management in it. Thus, in the ControlCenter4Kids internal tables only the number of each contact is stored. Whenever the remaining data is required, it is collected in the contacts framework of the Android platform.

\section{TECHNOLOGIES}

For the development of this project it was necessary to use a variety of development environments and technologies (Eclipse [17], Android SDK [18] and Android Development Tools [19]), a variety of components and frameworks of the Android platform (activities, services, content providers, broadcast receivers, SQLite, ...) and Android terminals (real and emulated).

\section{A. Android SDK}

The Android SDK (Software Development Kit) [18] framework includes a comprehensive set of development tools. These include a debugger, libraries, a terminal emulator, code examples and tutorials.

\section{B. Android Development Tools and Eclipse}

The Android Development Tools (ADT) [19] is a plugin for the officially supported development environment, Eclipse $I D E$ [17], which is designed to provide the programmer with a powerful, integrated environment for developing Android applications.

\section{Android Components}

We made use of all the basic components that Android offers, namely, activities, services, content providers and broadcast receivers [20].

The activity component is the base for all the screen interfaces that are used to present information and to perform the interactions with the user. It is the component that supports all the visible elements of the entire system.

The service component supports the background jobs. This component doesn't have an interface for the interaction with the user. In this project it is used for managing the device's location (GPS location) and sending it to the content provider responsible for this information.

The content providers are responsible for managing and providing information to other applications. In other words, they make data of a particular application visible to other applications. The way the data is stored is of the responsibility of whoever creates this component. In this system the data is stored using SQLite and regular files. It is used for storing and managing all the shared information like, contacts, information about messaging and calls made, GPS location, contacts allowed ...

The broadcast receiver is an implementation of the observer pattern that offers a client (application component) the possibility of being alerted about an event that has occurred. Each client registers into the system its intention to be informed about some events, to act accordingly. In this system we use broadcast receivers to be aware, for example, of all messages and calls that are made.

\section{D.SQLite}

As mentioned before, to store all the information that we need in this system we used regular files, stored into the memory card, but also traditional databases. For the database support, Android includes the SQLite subsystem. The programs that use the $S Q L i t e$ library can have access to a $S Q L$ database, without running a separate Relational DataBase Management (RDBM) process [21] [22].

The SQLite is not a client library used to connect to a large database server, but the server itself. The SQLite library writes and reads directly to and from the database on the device's memory.

\section{CONCLUSION}

Nowadays children obtain their first mobile phone at a younger age. There are multiple factors contributing to this trend. The main factor being that the parent wants to easily know what is happening with their children. Even being aware of the risks of having a smartphone at such a tender age parents offer it to their children. Due to this trend and the prices of these devices the development of this project focused on the Android platform.

Several hours were invested in the research and study of the internal features of the Android platform. A lot of time was also invested in the study of the ways to create user interfaces 
in order to improve the children's interaction in the Android platform. At the actual stage of the project it is possible to say that the Android platform is more secure and more appealing for our target audience. However, there are still some refinements to make in terms of graphics so that it can please the majority of our target audience. As the study and development of this system progressed on, it became clear that it would be an unfinished project. For instance, a feature that was not fully developed was the presentation of the various locations the child visited. Although we can see the various locations on GPS coordinates, this is not the most intuitive way to visualize the data. The ideal would be to create a file that can be exported and then presented, for example, in Google Maps, tracing the child's daily route. Another aim is also, in the long term, to develop a PC application to control and remotely customize the entire system. A set of improvements could also be convenient, because, where new communication technologies emerge, they always have to be analyzed and prepared in order to be child safe.

\section{REFERENCES}

[1] StatCounter, "Top 8 Mobile Operating Systems from December 2008 to December 2012," retrieved December 16, 2012, from "StatCounter Global Stats": http://gs.statcounter.com/\#mobile_os-ww-monthly200812-201212

[2] G. Stald and K. Ólafsson, "Mobile access: different users, different risks, different consequences?", in Children, Risk and Safety on the Internet: Research ans Policy Challenges in Comparative Prespective, S. Livingstone, L. Haddon and A. Gorzig, Eds. London:Policy Press, 2012, pp. 285-296.

[3] Google, "Google Play," retrieved December 16, 2012, from: http://play.google.com

[4] Android Does, "Apex Launcher," retrieved December 16, 2012, from "Google Play":

https://play.google.com/store/apps/details?id=com.anddoes.launcher

[5] AnderWeb, "ADW.Launcher," retrieved December 16, 2012, from "Google Play":

https://play.google.com/store/apps/details?id=org.adw.launcher

[6] Go Launcher Dev Team, "Go Launcher EX," retrieved December 16, 2012, from "Google Play":

https://play.google.com/store/apps/details?id=com.gau.go.launcherex

[7] TeslaCoila Software, "Nova Launcher," retrieved December 16, 2012 , from "Google Play":

https://play.google.com/store/apps/details?id=com.teslacoilsw.launcher

[8] CyanongenMod Team, "Trebuchet Launcher," retrieved December 16 2012, from "XDA-Developers": http://forum.xda-developers.com/show thread.php?t=1410674

[9] Kiddoware, "Kids Place - Parental Control," retrieved December 16, 2012, from "Google Play": https://play.google.com/store/apps/details?id $=$ com.kiddoware.kidsplace

[10] MMguardian.com, "MMGuardian ${ }^{\mathrm{TM}}$ Parental Control," retrieved December 16, 2012, from "Google Play": https://play.google.com/store/apps/details?id=com.teslacoilsw.launcher

[11] Smart App Cloud, "Android Parental Control," retrieved December 16, 2012, from "Google Play":

https://play.google.com/store/apps/details?id=com.vlobe.smartappcloud. android.parentalcontrol

[12] DoMobile Lab, "App Lock," retrieved December 16, 2012, from "Google Play":

https://play.google.com/store/apps/details?id=com.domobile.applock

[13] Jakar, "Missing Droid Locator," retrieved December 16, 2012, from "Google Play":

https://play.google.com/store/apps/details?id=com.jakar.missingdroid

[14] QAgate, "Track My Droid," retrieved December 16, 2012, from "Google Play":

https://play.google.com/store/apps/details?id=com.cgbcf
[15] handcent_market, "Handcent SMS," retrieved December 16, 2012, from "Google Play":

https://play.google.com/store/apps/details?id=com.handcent.nextsms

[16] Go Dev Team, "GO SMS Pro," retrieved December 16, 2012, from "Google Play":

https://play.google.com/store/apps/details?id=com.jb.gosms

[17] Eclipse Foundation, "Eclipse - The Eclipse Foundation open source community website," retrieved December 16, 2012, from: http://www.eclipse.org

[18] Google, "Android SDK," retrieved December 16, 2012, from "Android Developers": http://developer.android.com/sdk/index.html

[19] Google, "Installing the Eclipse Plugin," retrieved December 16, 2012, from "Android Developers": http://developer.android.com/sdk/installing/installing-adt.html

[20] Google, "Application Fundamentals," retrieved December 16, 2012, from "Android Developers": http://developer.android.com/guide/components/fundamentals.html

[21] Google, "Storage Options," retrieved December 16, 2012, from "Android Developers": http://developer.android.com/guide/topics/data/data-storage.html\#db

[22] Hipp, Wyrick \& Company, Inc., "About SQLite," retrieved December 16, 2012, from "SQLite": http://www.sqlite.org/about.html

\section{Creative Commons Attribution License 4.0 (Attribution 4.0 International, CC BY 4.0)}

This article is published under the terms of the Creative Commons Attribution License 4.0 https://creativecommons.org/licenses/by/4.0/deed.en_US 